\title{
Epigenetic regulation of long noncoding RNA UCA1 by SATB1 in breast cancer
}

\author{
Jong-Joo Lee ${ }^{1,2}$, Mikyoung Kim $^{1}$ E Hyoung-Pyo Kim ${ }^{1,2, *}$ \\ ${ }^{1}$ Department of Environmental Medical Biology, Institute of Tropical Medicine, Yonsei University College of Medicine, ${ }^{2}$ Brain Korea 21 \\ PLUS Project for Medical Science, Yonsei University College of Medicine, Seoul 03722, Korea
}

Special AT-rich sequence binding protein 1 (SATB1) is a nuclear matrix-associated DNA-binding protein that functions as a chromatin organizer. SATB1 is highly expressed in aggressive breast cancer cells and promotes growth and metastasis by reprograming gene expression. Through genomewide cross-examination of gene expression and histone methylation, we identified SATB1 target genes for which expression is associated with altered epigenetic marks. Among the identified genes, long noncoding RNA urothelial carcinoma-associated 1 (UCA1) was upregulated by SATB1 depletion. Upregulation of UCA1 coincided with increased H3K4 trimethylation (H3K4me3) levels and decreased H3K27 trimethylation (H3K27me3) levels. Our study showed that SATB1 binds to the upstream region of UCA1 in vivo, and that its promoter activity increases with SATB1 depletion. Furthermore, simultaneous depletion of SATB1 and UCA1 potentiated suppression of tumor growth and cell survival. Thus, SATB1 repressed the expression of oncogenic UCA1, suppressing growth and survival of breast cancer cells. [BMB Reports 2016; 49(10): 578-583]

\section{INTRODUCTION}

Breast cancer is the most frequently diagnosed life-threatening cancer in women and the leading cause of cancer death in American women after lung cancer (1). Formation and progression of breast cancer is driven by epigenetic alterations as well as progressive genetic abnormalities (2). Epigenetic alterations involve aberrations such as DNA methylation, histone modifications, and nucleosome remodeling. These epigenetic events modulate chromatin structure, which in turn

${ }^{*}$ Corresponding author. Tel: +82-2-2228-1842; Fax: +82-2-3638676; E-mail: kimhp@yuhs.ac

https://doi.org/10.5483/BMBRep.2016.49.10.156

Received 12 September 2016, Revised 20 September 2016, Accepted 22 September 2016

Keywords: Breast cancer, Epigenetic regulation, Histone methylation, SATB1, UCA1 causes aberrant transcriptional regulation that results in changes in the expression patterns of genes implicated in cellular proliferation, survival, and differentiation (3). Among epigenetic alterations, histone modifications play a fundamental role in carcinogenesis; recent advances in epigenomic analyses show that patterns of histone marks are profoundly altered in cancer cells (3).

Special AT-rich sequence binding protein 1 (SATB1) is a nuclear matrix-associated protein that binds to the ATC-rich DNA sequences of base unpairing regions (BURs) (4). Structurally, SATB1 consists of an N-terminal PDZ-like domain, a C-terminal homeodomain, and tandem CUT domains in the center (5). The PDZ-like domain contributes to the DNAbinding ability of SATB1 through oligomerization and modulates the association of SATB1 with other proteins via post-translational modifications. The CUT domains and the homeodomain mediate the sequence-specific binding of SATB1 to its DNA targets. Through BUR-binding, SATB1 tethers multiple genomic loci to the nuclear matrix to build the appropriate higher-order chromatin structure $(6,7)$. In addition, SATB1 recruits various transcription factors and chromatin modifying enzymes to regulate gene expression through histone modifications and nucleosomal remodeling at SATB1-bound matrix-associated regions (8). Therefore, SATB1 functions as a chromatin organizer that integrates global epigenetic and transcriptional programs, which are essential for cellular phenotypes and differentiation.

By controlling the expression of transcription factors such as GATA binding protein 3 (GATA3), Sfpi1, or Nanog, SATB1 functions in thymocyte development, $\mathrm{T}$ helper type 2 lineage commitment of naïve $\mathrm{T}$ cells, and embryonic stem cell differentiation $(9,10)$. Interestingly, high expression levels of SATB1 in metastatic breast cancer cells and in aggressive and poorly differentiated breast cancer tissues, as well as nonexpression (or non-detection) in normal adjacent tissues, suggest that SATB1 has a role in the metastatic phenotype of breast cancer cells (11). Depletion of SATB1 in aggressive breast cancer cells reverses the metastatic potential, whereas ectopic expression of SATB1 in non-aggressive cells induces metastatic and tumorigenic activities (11). These findings strongly suggest that SATB1 can function as a determinant of breast cancer metastasis. Moreover, a Kaplan-Meier survival

ISSN: 1976-670X (electronic edition)

Copyright (c) 2016 by the The Korean Society for Biochemistry and Molecular Biology

(c) This is an open-access article distributed under the terms of the Creative Commons Attribution Non-Commercial License (http://creativecommons.org/licenses/by-nc/4.0) which permits unrestricted non-commercial use, distribution, and reproduction in any medium, provided the original work is properly cited. 
analysis revealed a correlation between high expression levels of SATB1 with shorter survival rates in breast cancer patients, and a multivariate analysis confirmed that SATB1 is an independent prognostic marker in patients (12). Additional studies have associated SATB1 with the development of various cancers, including bladder, colorectal, gastric, liver, ovarian, pancreatic, and prostate cancer (13).

Although alterations in SATB1 expression are shown to disrupt the pattern and levels of histone acetylation, which promote tumor growth and metastasis through deregulation of gene expression (11), the effect of SATB1 on chromatin structure in a genome-wide scale, and its correlation with gene expression profiles have not been explored in breast cancer cells. In this study, we used chromatin immunoprecipitationcoupled to deep sequencing (ChIP-seq) to profile genomewide locations of $\mathrm{H} 3 \mathrm{~K} 4$ trimethylation (H3K4me3) and $\mathrm{H} 3 \mathrm{~K} 27$ trimethylation (H3K27me3) epigenetic marks in MDA-MB-231 aggressive breast cells. Furthermore, we identified a list of SATB1 target genes that showed significant alterations on these histone modifications after SATB1 depletion and examined their correlation with gene expression. Among the identified genes, we revealed that long noncoding RNA urothelial carcinoma-associated 1 (UCA1) was upregulated by SATB1 depletion and regulated via an epigenetic mechanism to control growth and apoptosis of aggressive breast cancer cells.

\section{RESULTS}

\section{Genome-wide changes in H3K4me3 and H3K27me3 levels by SATB1 depletion}

To investigate the role of SATB1 in tumor growth and metastasis in MDA-MB-231 breast cancer cells, we knocked down SATB1 expression by using lentiviral vector-mediated RNA interference of SATB1. Depletion of SATB1 in SATB1 short hairpin RNA (shRNA) (KD) cells was validated at messenger RNA (mRNA) and protein levels (Fig. S1A and S1B). We performed mRNA sequencing (mRNA-seq) using the SATB1-knockdown system and identified genes with significant change in expression levels (fold change $>2$, P value $<0.05$ ) (Fig. 1A). Differentially expressed genes (DEG) included 222 downregulated genes and 470 upregulated genes. To validate mRNA-seq data, we measured several altered genes by quantitative real-time polymerase chain reaction (qRT-PCR) (Fig. S1C). To assess whether SATB1 can affect genome-wide distribution of histone modification, we performed ChIP-seq using antibodies against $\mathrm{H} 3 \mathrm{~K} 4 \mathrm{me} 3$, which is present in promoters of actively transcribed genes, and against $\mathrm{H} 3 \mathrm{~K} 27 \mathrm{me} 3$, which is associated with the repression of transcription. ChIP-seq profiles in both control shRNA (CTL) and SATB1 shRNA (KD) cells revealed thousands of discrete genomic regions that are enriched with either $\mathrm{H} 3 \mathrm{~K} 4 \mathrm{me} 3$ or H3K27me3 marks. Consistent with a previous report (14), genomic regions with H3K4me3 or H3K27me3 modifications were identified preferentially at promoter or intergenic regions, respectively
(Fig. S2A).

The proximal sequences around the transcription start site (TSS) are essential elements of gene regulation (15). Therefore, we examined uniquely mapped tags of $\mathrm{H} 3 \mathrm{~K} 4 \mathrm{me} 3$ and H3K27me3 at promoter regions, which were defined in this study as $3.0 \mathrm{~kb}$ upstream and downstream of the TSS $( \pm 3.0$ $\mathrm{kb}$ around the TSS). According to the estimation provided by mRNA-seq analysis, highly transcribed genes exhibited high H3K4me3 levels but very low H3K27me3 levels around the TSS. In contrast, silent genes were depleted of $\mathrm{H} 3 \mathrm{~K} 4 \mathrm{me} 3$ marks and exhibited high levels of H3K27me3 (Fig. S2B and $\mathrm{S} 2 \mathrm{C})$. These results confirmed the positive correlation between transcription activity and $\mathrm{H} 3 \mathrm{~K} 4 \mathrm{me} 3$ levels at promoter regions, and the negative correlation between transcription activity and H3K27me3 levels at promoter regions. To investigate potential changes in H3K4me3 and H3K27me3 levels caused by SATB1 depletion, we compared genome-wide enrichment of $\mathrm{H} 3 \mathrm{~K} 4 \mathrm{me} 3$

A

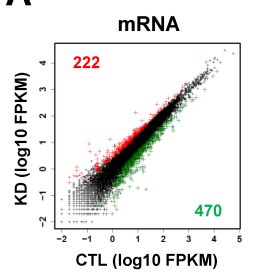

B

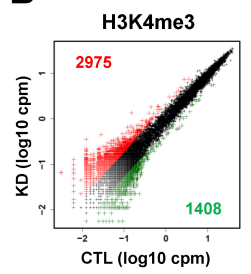

C

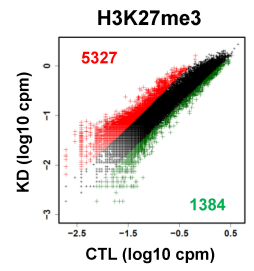

D

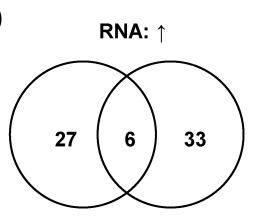

H3K4me3: $\uparrow$ H3K27me3 : $\downarrow$

E

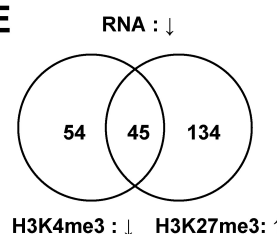

Fig. 1. Genome-wide changes in gene expression and histone methylation induced by SATB1 depletion. MDA-MB-231 cells were infected with lentiviruses expressing shRNA against SATB1 or containing empty pLKO.1 vector; drug-resistant cells were selected. (A) Scatter plot shows differentially expressed genes from control shRNA (CTL) and SATB1 shRNA (KD) cells. Significantly changed genes (fold change $>2, \mathrm{P}$ value $<0.05$ ) that are upregulated in SATB1 shRNA cells (red) or downregulated in SATB1 shRNA cells (green) are indicated. (B and $\mathrm{C}$ ) Scatter plots show genes with differential enrichment of $\mathrm{H} 3 \mathrm{~K} 4 \mathrm{me} 3$ (B) and H3K27me3 (C) within the promoter region (3 $\mathrm{kb}$ either side of the TSS) for control shRNA (CTL) and SATB1 shRNA (KD) cells. Significantly changed genes (fold change $>1.5, \mathrm{P}$ value $<0.05$ ) that are upregulated in SATB1 shRNA cells (red) or downregulated in SATB1 shRNA cells (green) are indicated. (D) Venn diagram shows overlap of SATB1 knockdown-induced genes that were upregulated in RNA expression, had greater H3K4me3 enrichment at the promoter region, and had lesser $\mathrm{H} 3 \mathrm{~K} 27 \mathrm{me} 3$ enrichment at the promoter region. (E) Venn diagram shows overlap of SATB1 knockdowninduced genes that were downregulated in RNA expression, had lesser enrichment with $\mathrm{H} 3 \mathrm{~K} 4 \mathrm{me} 3$ at the promoter region, and had greater enrichment with $\mathrm{H} 3 \mathrm{~K} 27 \mathrm{me} 3$ at the promoter region. 
and H3K27me3 at gene promoter regions for control shRNA (CTL) and SATB1 shRNA (KD) cells. By using the edgeR (empirical analysis of digital gene expression data in R) approach, we identified 2,975 and 1,408 gene promoter regions with differentially higher and lower levels (fold change $>1.5)$, respectively, of H3K4me3 in SATB1-knockdown cells (Fig. 1B). We also identified 5,327 and 1,384 gene promoter regions with differentially higher and lower levels of H3K27me3 in SATB1-knockdown cells (fold change > 1.5), respectively. (Fig. 1C). Next, we focused on DEGs to identify SATB1 target genes for which expressions are regulated by altered histone methylation. Among the 222 genes upregulated by SATB1 depletion, we identified 33 genes with higher H3K4me3 levels, 39 genes with lower H3K27me3 levels, and 6 genes with both higher $\mathrm{H} 3 \mathrm{~K} 4 \mathrm{me} 3$ and lower H3K27me3 levels (Fig. 1D, Table S2). Among the 470 genes downregulated by SATB1 depletion, we found 99 genes with lower H3K4me3 levels, 179 genes with higher H3K27me3 levels, and 45 genes with both lower H3K4me3 and higher H3K27me3 levels (Fig. 1E, Table S3).

\section{Repression of long noncoding RNA UCA1 by SATB1}

We identified long noncoding RNA (IncRNA) urothelial carcinoma-associated 1 (UCA1) as one of the epigenetically regulated SATB1 target genes (Fig. 2A). UCA1 was originally identified in bladder transitional cell carcinoma (16) and is known to play an important role in the occurrence and development of many tumor and non-tumor diseases (17). Integration of mRNA-seq and ChIP-seq demonstrated that
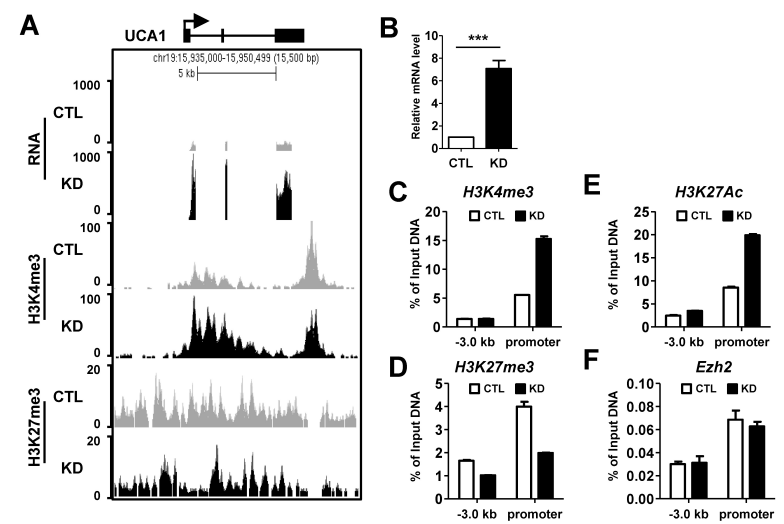

Fig. 2. Upregulation of UCA1 induced by SATB1 depletion coincides with altered histone methylation. (A) Genomic snapshot of the UCA1 locus. Density of mRNA-seq reads and ChIP-seq reads of $\mathrm{H} 3 \mathrm{~K} 4 \mathrm{me} 3$ and $\mathrm{H} 3 \mathrm{~K} 27 \mathrm{me} 3$ in control shRNA (CTL) and SATB1 shRNA (KD) cells are shown. (B) qRT-PCR validation of UCA1 expression in control shRNA (CTL) and SATB1 shRNA (KD) cells (mean \pm standard error of the mean [SEM] of three independent biological replicates) (C-F) ChIP-qPCR validation of H3K4me3 (C), H3K27me3 (D), H3K27Ac (E), and EZH2 (F) levels at the UCA1 locus. upregulation of UCA1 IncRNA after SATB1 depletion coincided with an increased level of H3K4me3 and a decreased level of H3K27me3 at the promoter (Fig. 2A). These results were validated independently by performing qRT-PCR (Fig. 2B) and ChIP-qPCR (Fig. 2C and 2D). An increased level of H3K27 acetylation (H3K27Ac), another active gene regulatory histone modification marker, at UCA1 promoter region was also increased by SATB1 depletion (Fig. 2E). However, the enrichment of Ezh2, which catalyzes trimethylation of $\mathrm{H} 3 \mathrm{~K} 27$, was not changed at the UCA1 promoter region (Fig. 2F).

To further understand the molecular basis of SATB1-mediated UCA1 repression, we examined whether UCA1 is directly regulated by SATB1. Analysis of ChIP-seq data against SATB1 (unpublished data from our laboratory) revealed prominent SATB1 binding to the promoter and the 3.0-kb upstream region of UCA1 (Fig. 3A). Binding of SATB1 to these regions was independently validated by performing ChIP-qPCR (Fig. 3B). Furthermore, SATB1 depletion abrogated the enrichment of SATB1 on the UCA1 locus, further confirming the validity of the ChIP-seq data (Fig. 3B). To test whether the promoter and the 3.0-kb upstream region of UCA1 contain SATB1 response elements, the regions were separately subcloned into a luciferase reporter construct. Transfection of these luciferase constructs into MDA-MB-231 cells revealed a significant increase of promoter activities by SATB1 depletion (Fig. 3C). The data indicated that SATB1 represses UCA1 expression by directly binding to the UCA1 promoter and 3.0-kb upstream regions.

In contrast to the findings for SATB1-mediated UCA1 repression, SATB1 expression remained unaltered in MDAMB-231 cells expressing shRNA against UCA1, which suggests that SATB1 is the upstream regulator of UCA1 expression (Fig.

A

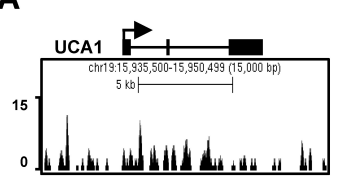

B

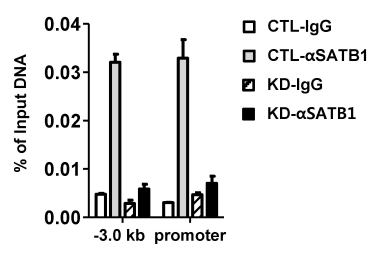

C

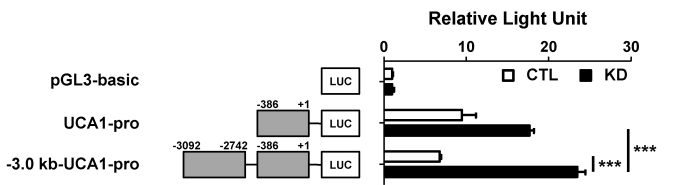

Fig. 3. Direct regulation of UCA1 by SATB1. (A) Genomic snapshot of the UCA1 locus showing ChIP-seq reads for SATB1 in MDA-MB-231 cells. (B) ChIP-qPCR validation for SATB1 binding at the UCA1 locus in control shRNA (CTL) and SATB1 shRNA (KD) cells. (C) Luciferase constructs containing UCA1 promoter with or without 3.0-kb upstream region were transfected into control shRNA (CTL) and SATB1 shRNA (KD) cells. Values are mean \pm SEM of results from three experiments. 
A

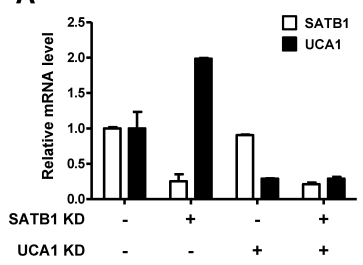

B

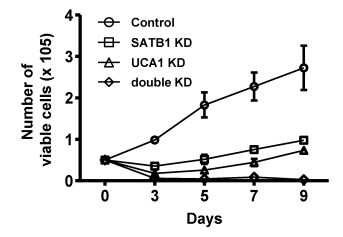

C

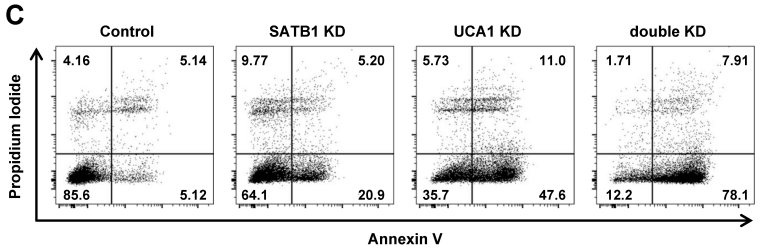

Fig. 4. Effect of SATB 1 and UCA1 on breast cancer cell growth and apoptosis. MDA-MB-231 cells were infected with lentiviruses expressing shRNA against SATB1 or UCA1; drug-resistant cells were selected. (A) qRT-PCR was performed to analyze mRNA levels of SATB1 and UCA1 and normalized against 18S ribosomal RNA (rRNA) levels. (B) Viable cells were counted by Trypan blue staining after depletion of SATB1 and/or UCA1. (C) Flow cytometric analysis of apoptotic cells induced by depletion of SATB1 and/or UCA1. For each sample, the bar indicates standard deviation (SD) from three experiments.

4A). MDA-MB-231 cells simultaneously infected with lentiviruses expressing shRNA against SATB1 and UCA1, showed a significant decrease of expression for both genes (Fig. 4A). In agreement with previous reports $(11,18)$, depletion of either SATB1 or UCA1 suppressed the growth of MDA-MB-231 cells (Fig. 4B), which supports the oncogenic roles of both genes. Interestingly, cell viability was nearly abrogated by simultaneous depletion of SATB1 and UCA1 (Fig. 4B). In addition, we analyzed apoptosis of MDA-MB-231 cells after knockdown of SATB1 or UCA1 expression. Depletion of either SATB1 or UCA1 triggered significant apoptosis of MDA-MB-231 cells, and simultaneous depletion of both genes further increased apoptosis (Fig. 4C). The data indicated that SATB1 depletion results in upregulation of oncogenic IncRNA UCA1, which promotes growth and survival of MDA-MB-231 breast cancer cells.

\section{DISCUSSION}

SATB1 is a global chromatin organizer that integrates higherorder chromatin architecture with regulation of gene expression and modulates the accessibility of many gene loci to chromatin remodeling enzymes and transcription factors. Several studies have demonstrated the critical role of SATB1 in cancer in which enhanced expression of SATB1 promotes aberrant growth and metastasis of various epithelial tumors by reprogramming global transcriptional profiles. In this study, we investigated the effect of SATB1 on histone modification status in a genome-wide scale to better understand its epigenetic mechanism in breast cancer. Two histone methylation marks, activating $\mathrm{H} 3 \mathrm{~K} 4 \mathrm{me} 3$ and silencing $\mathrm{H} 3 \mathrm{~K} 27 \mathrm{me} 3$, were analyzed by ChIP-seq. We focused on the promoter regions for the enrichment of these epigenetic marks, and thousands of genes were found to have differential levels of H3K4me3 and H3K27me3 after SATB1 depletion. These changes in H3K4me3 and H3K27me3 levels support the suggested role of SATB1 as a global chromatin organizer and epigenetic factor. By integrating chromatin modification (ChIP-seq) with gene expression (mRNA-seq), we identified SATB1 target genes for which expression is associated with histone modifications. Depletion of SATB1 led to upregulation of genes that coincided with higher H3K4me3 levels and lower H3K27me3 levels at promoter regions. In addition, SATB1 depletion resulted in downregulation of genes for which expression was associated with lower H3K4me3 levels and higher H3K27me3 levels. However, many genes differentially expressed by SATB1 depletion did not show significantly altered histone modifications at their promoters. SATB1 may regulate the expression of these genes by modulating chromatin structure at distal regulatory elements other than the promoter region, or by mediating long-range chromatin interaction between such regulatory elements and their cognate promoters.

Here, we revealed that UCA1 is a novel SATB1 target gene, which is regulated via an epigenetic mechanism in aggressive breast cancer cells. UCA1 is an IncRNA that is aberrantly expressed in a broad range of cancers and plays oncogenic roles in tumor growth and metastasis. Recent studies show that UCA1 expression can be upregulated by several transcription factors, including Ets-2, C/EBP $\alpha$, and HIF-1 $\alpha$ (19-21). In addition, transforming growth factor beta (TGF- $\beta$ ) treatment of breast cancer cells is reported to induce UCA1 expression by recruiting a transcriptional complex composed of TAZ, YAP, TEAD and SMAD2/3 (22). In contrast, repression of UCA1 expression is mediated in human foreskin fibroblasts by CAPER $\alpha /$ TBX3 transcriptional complex (23).

Previous studies have focused on the role of SATB1 in mRNA expression but its role in IncRNA transcription has not been studied. This study seems to be the first report describing the function of SATB1 in IncRNA transcription. In addition to UCA1, RNA-seq analysis revealed that expression of other IncRNAs, such as HCP5 and LOC100506844, are positively regulated by SATB1. Further studies will be necessary to dissect how these IncRNAs are regulated by SATB1.

Upregulation of UCA1 after SATB1 depletion coincided with increased levels of activating histone marks, H3K4me3 and $\mathrm{H} 3 \mathrm{~K} 27 \mathrm{Ac}$, and a decreased level of repressive H3K27me3 at the promoter. This result suggests that SATB1 represses UCA1 expression by closing the chromatin structure at the promoter region of UCA1. ChIP assay showed in vivo occupancy of SATB1 at the promoter and 3.0-kb upstream regions of UCA1. Moreover, transient transfection experiments using luciferase reporter constructs confirmed the repressive activity of these 
SATB1 binding sites. Because enrichment of the histone methyltransferase EZH2 at the UCA1 promoter region was not affected by SATB1 depletion, the change in H3K27me3 level at the promoter may not be dependent on EZH2. H3K27 demethylases UTX and JMJD3 has been discovered to actively demethylate H3K27me3 (24), and UTX has been shown to be part of the H3K4me3 methyltransferase complex (25). Whether SATB1 can affect the recruitment of these H3K27 demethylases and $\mathrm{H} 3 \mathrm{~K} 4 \mathrm{me} 3$ methyltransferase complex to the UCA1 locus remains to be elucidated.

The role of UCA1 in cell proliferation and apoptosis has been studied in various cancers. In breast cancer cells, UCA1 interacts with heterogeneous nuclear ribonucleoprotein I (hnRNP I) and suppresses p27 protein expression, leading to increased cell proliferation (18). In addition, UCA1 interacts directly with miR-143 and modulates breast cancer cell growth and apoptosis (26). Consistent with those reports, our study indicated that depletion of UCA1 results in attenuated growth and induced apoptosis in MDA-MB-231 cells. Depletion of SATB1 resulted in induced UCA1 expression, and simultaneous depletion of SATB1 and UCA1 potentiated the suppression of growth and survival of breast cancer cells. These effects indicate that upregulated UCA1 may partially rescue the growth and survival of SATB1-knockdown MDA-MB-231 cells. Our results strongly suggest that targeting SATB1 by itself will not be sufficient to treat breast cancer, but may require combination strategies of anti-SATB1 with gene targets such as UCA1.

\section{MATERIALS AND METHODS}

Detailed experimental procedures are described in Supplementary Information.

\section{ACKNOWLEDGEMENTS}

This work was supported by a grant from the National R\&D Program for Cancer Control, Ministry for Health, Welfare and Family Affairs, Korea (1120370 to H.-P. Kim), and a National Research Foundation (NRF) of Korea grant (MEST; NRF-20110030086, 2012M3A9B4028272, and 2016R1A2B4014183 to H.-P. Kim).

\section{REFERENCES}

1. Jemal A, Bray F, Center MM, Ferlay J, Ward E and Forman D (2011) Global cancer statistics. CA Cancer J Clin 61, 69-90

2. Hanahan D and Weinberg RA (2011) Hallmarks of cancer: the next generation. Cell 144, 646-674

3. Allis CD and Jenuwein T (2016) The molecular hallmarks of epigenetic control. Nat Rev Genet 17, 487-500

4. Dickinson LA, Joh T, Kohwi $Y$ and Kohwi-Shigematsu $T$ (1992) A tissue-specific MAR/SAR DNA-binding protein with unusual binding site recognition. Cell 70, 631-645

5. Wang Z, Yang X, Chu X et al (2012) The structural basis for the oligomerization of the $\mathrm{N}$-terminal domain of SATB1. Nucleic Acids Res 40, 4193-4202

6. Kumar PP, Bischof O, Purbey PK et al (2007) Functional interaction between PML and SATB1 regulates chromatinloop architecture and transcription of the MHC class I locus. Nat Cell Biol 9, 45-56

7. Cai S, Han HJ and Kohwi-Shigematsu T (2003) Tissuespecific nuclear architecture and gene expression regulated by SATB1. Nat Genet 34, 42-51

8. Yasui D, Miyano M, Cai S, Varga-Weisz P and KohwiShigematsu T (2002) SATB1 targets chromatin remodelling to regulate genes over long distances. Nature 419, 641-645

9. Burute M, Gottimukkala K and Galande S (2012) Chromatin organizer SATB1 is an important determinant of T-cell differentiation. Immunol Cell Biol 90, 852-859

10. Savarese F, Davila A, Nechanitzky R et al (2009) Satb1 and Satb2 regulate embryonic stem cell differentiation and Nanog expression. Genes Dev 23, 2625-2638

11. Han HJ, Russo J, Kohwi Y and Kohwi-Shigematsu T (2008) SATB1 reprogrammes gene expression to promote breast tumour growth and metastasis. Nature 452, 187-193

12. Kohwi-Shigematsu T, Poterlowicz K, Ordinario E, Han HJ, Botchkarev VA and Kohwi Y (2013) Genome organizing function of SATB1 in tumor progression. Semin Cancer Biol 23, 72-79

13. Mir R, Pradhan SJ and Galande S (2012) Chromatin organizer SATB1 as a novel molecular target for cancer therapy. Curr Drug Targets 13, 1603-1615

14. Barski A, Cuddapah S, Cui K et al (2007) High-resolution profiling of histone methylations in the human genome. Cell 129, 823-837

15. Rosenbloom KR, Dreszer TR, Long JC et al (2012) ENCODE whole-genome data in the UCSC Genome Browser: update 2012. Nucleic Acids Res 40, D912-917

16. Wang F, Li X, Xie X, Zhao L and Chen W (2008) UCA1, a non-protein-coding RNA up-regulated in bladder carcinoma and embryo, influencing cell growth and promoting invasion. FEBS Lett 582, 1919-1927

17. Xue $M$, Chen $W$ and Li X (2015) Urothelial cancer associated 1: a long noncoding RNA with a crucial role in cancer. J Cancer Res Clin Oncol 142, 1407-1419

18. Huang J, Zhou N, Watabe K et al (2014) Long non-coding RNA UCA1 promotes breast tumor growth by suppression of p27 (Kip1). Cell Death Dis 5, e1008

19. Wu W, Zhang S, Li X, Xue M, Cao S and Chen W (2013) Ets-2 regulates cell apoptosis via the Akt pathway, through the regulation of urothelial cancer associated 1, a long non-coding RNA, in bladder cancer cells. PLoS One 8, e73920

20. Xue M, Li X, Wu W et al (2014) Upregulation of long non-coding RNA urothelial carcinoma associated 1 by CCAAT/enhancer binding protein alpha contributes to bladder cancer cell growth and reduced apoptosis. Oncol Rep 31, 1993-2000

21. Xue M, Li X, Li Z and Chen W (2014) Urothelial carcinoma associated 1 is a hypoxia-inducible factor1alpha-targeted long noncoding RNA that enhances hypoxic bladder cancer cell proliferation, migration, and invasion. Tumour Biol 35, 6901-6912 
22. Hiemer SE, Szymaniak AD and Varelas X (2014) The transcriptional regulators TAZ and YAP direct transforming growth factor beta-induced tumorigenic phenotypes in breast cancer cells. J Biol Chem 289, 13461-13474

23. Kumar PP, Emechebe U, Smith R et al (2014) Coordinated control of senescence by IncRNA and a novel T-box3 co-repressor complex. Elife 3 e02805

24. Agger K, Cloos PA, Christensen J et al (2007) UTX and JMJD3 are histone H3K27 demethylases involved in HOX gene regulation and development. Nature 449, 731-734

25. Issaeva I, Zonis Y, Rozovskaia T et al (2007) Knockdown of ALR (MLL2) reveals ALR target genes and leads to alterations in cell adhesion and growth. Mol Cell Biol 27, 1889-1903

26. Tuo YL, Li XM and Luo J (2015) Long noncoding RNA UCA1 modulates breast cancer cell growth and apoptosis through decreasing tumor suppressive miR-143. Eur Rev Med Pharmacol Sci 19, 3403-3411 